\title{
The instantaneous fluctuation theorem
}

\author{
Charlotte F. Petersen, ${ }^{\text {a) }}$ Denis J. Evans, and Stephen R. Williams ${ }^{\text {b) }}$ \\ Research School of Chemistry, Australian National University, Canberra, ACT 0200, Australia
}

(Received 28 August 2013; accepted 23 October 2013; published online 11 November 2013)

\begin{abstract}
We give a derivation of a new instantaneous fluctuation relation for an arbitrary phase function which is odd under time reversal. The form of this new relation is not obvious, and involves observing the system along its transient phase space trajectory both before and after the point in time at which the fluctuations are being compared. We demonstrate this relation computationally for a number of phase functions in a shear flow system and show that this non-locality in time is an essential component of the instantaneous fluctuation theorem. () 2013 AIP Publishing LLC. [http://dx.doi.org/10.1063/1.4829445]
\end{abstract}

\section{INTRODUCTION}

Fluctuation relations typically provide information about path integrals of phase functions in time-reversible dynamical systems. ${ }^{1-16}$ In this paper we will derive a fluctuation relation that gives the relative probability that the instantaneous values of a phase function take on opposite values, $\pm A$, in terms of the well known path integral of the dissipation function.

The derivation of the new theorem rests on a key result by Evans et al. ${ }^{17}$ which gives the form of the covariant dissipation function. We will provide a brief summary of the relevant result here. The dissipation function is one of the most important functions in non-equilibrium statistical mechanics, and is the argument of the Evans-Searles fluctuation theorem, ${ }^{18}$ the dissipation theorem, ${ }^{19}$ and the equilibrium relaxation theorems. ${ }^{20}$ It is a function of the dynamics of the system and a distribution function. Typically the distribution function used is the known initial distribution of the system. The covariant dissipation function is defined with respect to the time evolving distribution function as the system relaxes. The new result allows us to interconvert the usual dissipation function with the time covariant dissipation function.

To represent this situation exactly, we need to use more detailed notation than what has typically been used in the past. The distribution function used will be defined by the point in time, $t_{b}$, at which the system takes on this distribution. To fully specify the dissipation function we also need the variables $\tau$, the time interval the dissipation function is integrated over, and $t_{a}$, the starting point of this interval. We assume the dynamics is autonomous. We will use the notation set out in the previous work ${ }^{17}$ for the integrated dissipation function

$$
\begin{aligned}
\Omega_{\tau}\left(S^{t_{a}} \boldsymbol{\Gamma} ; t_{b}\right) & \equiv \int_{t_{a}}^{t_{a}+\tau} \Omega\left(S^{s} \boldsymbol{\Gamma} ; t_{b}\right) d s \\
& \equiv \ln \left(\frac{f\left(S^{t_{a}} \boldsymbol{\Gamma} ; t_{b}\right)}{f\left(M^{T} S^{\tau} S^{t_{a}} \boldsymbol{\Gamma} ; t_{b}\right)}\right)-\int_{t_{a}}^{t_{a}+\tau} \Lambda\left(S^{s} \boldsymbol{\Gamma}\right) d s,
\end{aligned}
$$

\footnotetext{
${ }^{a)}$ Electronic mail: charlotte.petersen@anu.edu.au

b) Electronic mail: swilliams@ rsc.anu.edu.au
}

where $\Gamma$ is the phase space position at time $0, S^{t}$ is the phase space evolution operator acting for a time $t, M^{T}$ is the time reversal operator, $\Omega(\boldsymbol{\Gamma} ; t)$ is the instantaneous dissipation function defined with respect to the distribution function at time $t, f(\boldsymbol{\Gamma} ; t)$ is the phase space density at position $\Gamma$ for the distribution function of the system at time $t$, and $\Lambda$ is the phase space expansion factor. Because the dynamics is autonomous, $\dot{\boldsymbol{\Gamma}}=\dot{\boldsymbol{\Gamma}}(\boldsymbol{\Gamma})$ is not an explicit function of both phase and time and likewise the phase space expansion factor $\Lambda=\Lambda(\boldsymbol{\Gamma}) \equiv \partial / \partial \boldsymbol{\Gamma} \cdot \dot{\boldsymbol{\Gamma}}$.

Using the dissipation theorem ${ }^{19}$ and the definition of the dissipation function, Eq. (1), the following relationship between the standard and covariant dissipation function was derived: ${ }^{17}$

$$
\Omega_{\tau}\left(S^{t_{a}} \boldsymbol{\Gamma} ; t_{a}\right)=\Omega_{2 t_{a}+\tau}(\boldsymbol{\Gamma} ; 0) .
$$

The covariant dissipation function, defined with respect to the distribution function at time $t_{a}$ and integrated over the time interval $\left(t_{a}, t_{a}+\tau\right)$ is equal to the dissipation function defined from the distribution function at time 0 , integrated over the interval $\left(0,2 t_{a}+\tau\right)$. The covariant dissipation function is not local in time. Taking the case where $\tau=0$, Eq. (2) becomes

$$
\Omega_{0}\left(S^{t_{a}} \boldsymbol{\Gamma} ; t_{a}\right)=\Omega_{2 t_{a}}(\boldsymbol{\Gamma} ; 0),
$$

which demonstrates that while the distribution function is even in the momenta in equilibrium initially, it is not at later times. This can be written as $f(\boldsymbol{\Gamma} ; 0)=f\left(M^{T} \boldsymbol{\Gamma} ; 0\right)$ in equilibrium, but $f\left(S^{t_{a}} \boldsymbol{\Gamma} ; t_{a}\right) \neq f\left(M^{T} S^{t_{a}} \boldsymbol{\Gamma} ; t_{a}\right)$.

\section{THEORY}

To derive the new relation we consider a deterministic system initially at equilibrium at time 0 that then undergoes a transient when a constant force is applied. We are interested in the instantaneous probability of a phase function at some particular time, $t_{1}$, during this transient.

The Evans-Searles transient fluctuation relation ${ }^{21}$ involves a quantity now known as dissipation. The fluctuation theorem (FT) gives the probability of observing a set of trajectories in some small volume of phase space $\delta V_{\Gamma}$ centered on some phase space vector compared to the conjugate set of 
anti-trajectories

$$
\frac{\operatorname{Pr}\left(\delta V_{\boldsymbol{\Gamma}}\left(S^{t_{1}} \boldsymbol{\Gamma}\right) ; t_{1}\right)}{\operatorname{Pr}\left(\delta V_{\boldsymbol{\Gamma}}\left(M^{T} S^{\tau} S^{t_{1}} \boldsymbol{\Gamma}\right) ; t_{1}\right)}=\exp \left[\Omega_{\tau}\left(S^{t_{1}} \boldsymbol{\Gamma} ; t_{1}\right)\right]
$$

where $S^{t_{1}}$ is the natural time evolution operator, $M^{T}(\mathbf{q}, \mathbf{p})$ $\equiv(\mathbf{q},-\mathbf{p})$ is the time reversal mapping, ${ }^{22} \delta V_{\boldsymbol{\Gamma}}\left(S^{t_{1}} \boldsymbol{\Gamma}\right)$ is the infinitesimal volume around the phase space position $S^{t_{1}} \boldsymbol{\Gamma}$, and $\operatorname{Pr}\left(\delta V_{\boldsymbol{\Gamma}}\left(S^{t_{1}} \boldsymbol{\Gamma}\right) ; t_{1}\right)$ is the probability of observing ensemble members inside this volume at time $t_{1}$. If we consider a trajectory of length $\tau=0$, Eq. (4) becomes

$$
\frac{\operatorname{Pr}\left(\delta V_{\boldsymbol{\Gamma}}\left(S^{t_{1}} \boldsymbol{\Gamma}\right) ; t_{1}\right)}{\operatorname{Pr}\left(\delta V_{\boldsymbol{\Gamma}}\left(M^{T} S^{t_{1}} \boldsymbol{\Gamma}\right) ; t_{1}\right)}=\exp \left[\Omega_{0}\left(S^{t_{1}} \boldsymbol{\Gamma} ; t_{1}\right)\right]
$$

In this special case the conjugate anti-trajectory begins from the time reversed map of the starting point of the forward trajectory. In the past we have usually been interested in time averages of dissipation. Equation (5) does not involve a time average because the duration of the path integral is 0 .

In Eq. (5), $\Omega_{0}\left(S^{t_{1}} \boldsymbol{\Gamma} ; t_{1}\right)$ is defined with respect to the distribution at a time $t_{1}$. Using Eq. (3), the key result from Evans et $a l .{ }^{17}$ summarised in the Introduction, we can rewrite the covariant dissipation function in a form that is easier to deal with,

$$
\frac{\operatorname{Pr}\left(\delta V_{\boldsymbol{\Gamma}}\left(S^{t_{1}} \boldsymbol{\Gamma}\right) ; t_{1}\right)}{\operatorname{Pr}\left(\delta V_{\boldsymbol{\Gamma}}\left(M^{T} S^{t_{1}} \boldsymbol{\Gamma}\right) ; t_{1}\right)}=\exp \left[\Omega_{2 t_{1}}(\boldsymbol{\Gamma} ; 0)\right]
$$

It is now defined with respect to the distribution function at time $t=0$ and the length of the trajectory is $2 t_{1}$. The equality of (5) and (6) is easily seen by combining time reversibility with the conservation of probabilities. Those ensemble members that are located within $\delta V_{\boldsymbol{\Gamma}}(\boldsymbol{\Gamma})$ at $t=0$ also flow through $\delta V_{\boldsymbol{\Gamma}}\left(S^{t_{1}} \boldsymbol{\Gamma}\right)$ at time $t_{1}$, and through $\delta V_{\boldsymbol{\Gamma}}\left(S^{2 t_{1}} \boldsymbol{\Gamma}\right)$ at time $2 t_{1}$.

Let us consider an arbitrary phase function, $B\left(S^{t} \Gamma\right)$, that is odd under a time reversal transformation. We can write the probability of particular values of the phase function, $B\left(S^{t} \boldsymbol{\Gamma}\right)=A$, in terms of the probability of infinitesimal volumes around particular phase space positions. Summing each of these volumes gives the relative probability of such values,

$$
\frac{\operatorname{Pr}\left(B\left(S^{t_{1}} \boldsymbol{\Gamma}\right)=A\right)}{\operatorname{Pr}\left(B\left(S^{t_{1}} \boldsymbol{\Gamma}\right)=-A\right)}=\frac{\sum_{\left.\delta V_{\boldsymbol{\Gamma}}\left(S^{t_{1}} \boldsymbol{\Gamma}\right)\right|_{B=A}} \operatorname{Pr}\left(\delta V_{\boldsymbol{\Gamma}}\left(S^{t_{1}} \boldsymbol{\Gamma}\right) ; t_{1}\right)}{\sum_{\left.\delta V_{\boldsymbol{\Gamma}}\left(S^{t_{1}} \boldsymbol{\Gamma}\right)\right|_{B=-A}} \operatorname{Pr}\left(\delta V_{\boldsymbol{\Gamma}}\left(S^{t_{1}} \boldsymbol{\Gamma}\right) ; t_{1}\right)}
$$

Substituting in (6) gives

$$
\begin{aligned}
& \frac{\operatorname{Pr}\left(B\left(S^{t_{1}} \boldsymbol{\Gamma}\right)=A\right)}{\operatorname{Pr}\left(B\left(S^{t_{1}} \boldsymbol{\Gamma}\right)=-A\right)} \\
& \quad=\frac{\sum_{\left.\delta V_{\boldsymbol{\Gamma}}\left(S^{t_{1}} \boldsymbol{\Gamma}\right)\right|_{B=A}} \operatorname{Pr}\left(\delta V_{\boldsymbol{\Gamma}}\left(S^{t_{1}} \boldsymbol{\Gamma}\right) ; t_{1}\right)}{\sum_{\left.\delta V_{\Gamma}\left(S^{t_{1}} \boldsymbol{\Gamma}\right)\right|_{B=-A}} \exp \left[\Omega_{2 t_{1}}(\boldsymbol{\Gamma} ; 0)\right] \operatorname{Pr}\left(\delta V_{\boldsymbol{\Gamma}}\left(M^{T} S^{t_{1}} \boldsymbol{\Gamma}\right) ; t_{1}\right)} .
\end{aligned}
$$

To understand this sum over volumes defined by phase space points $S^{t_{1}} \boldsymbol{\Gamma}$, where the probability in the argument is defined by the time reverse map of these points, $M^{T} S^{t_{1}} \Gamma$, we need to understand the relationship between the trajectory and antitrajectory pair. This is represented visually in Figure 1.

It is easy to see from Figure 1 why the dissipation function needs to be defined over the time interval 0 to $2 t_{1}$; at the midpoint of the interval the position in phase space that the trajectory has evolved to is the time reverse map of the position reached by the antitrajectory. Since $B(\boldsymbol{\Gamma})$ is odd under the time reversal transformation, for each trajectory where $B\left(S^{t_{1}} \boldsymbol{\Gamma}\right)=-A$, the antitrajectory has $B\left(M^{T} S^{t_{1}} \boldsymbol{\Gamma}\right)$ $=B\left(S^{t_{1}} M^{T} S^{2 t_{1}} \Gamma\right)=A$. This allows us to rewrite the denominator of Eq. (8) in terms of the following conjugate antitrajectory volumes:

$$
\frac{\operatorname{Pr}\left(B\left(\boldsymbol{\Gamma}\left(t_{1}\right)\right)=A\right)}{\operatorname{Pr}\left(B\left(\boldsymbol{\Gamma}\left(t_{1}\right)\right)=-A\right)}=\frac{\sum_{\left.\delta V_{\boldsymbol{\Gamma}}\left(S^{t_{1}} \boldsymbol{\Gamma}\right)\right|_{B=A}} \operatorname{Pr}\left(\delta V_{\boldsymbol{\Gamma}}\left(S^{t_{1}} \boldsymbol{\Gamma}\right) ; t_{1}\right)}{\sum_{\left.\delta V_{\boldsymbol{\Gamma}}\left(S^{t_{1}} M^{T} S_{2}^{t_{2}} \boldsymbol{\Gamma}\right)\right|_{B=A}} \exp \left[-\Omega_{2 t_{1}}\left(M^{T} S^{2 t_{1}} \boldsymbol{\Gamma} ; 0\right)\right] \operatorname{Pr}\left(\delta V_{\boldsymbol{\Gamma}}\left(S^{t_{1}} M^{T} S^{2 t_{1}} \boldsymbol{\Gamma}\right) ; t_{1}\right)} .
$$

Here we have also used the definition of the dissipation function ${ }^{1}$ to replace $\exp \left[\Omega_{2 t_{1}}(\boldsymbol{\Gamma} ; 0)\right]$ with $\exp \left[-\Omega_{2 t_{1}}\left(M^{T} S^{2 t_{1}} \Gamma ; 0\right)\right]$. The initial distribution function is always assumed to be even in the momenta.

Recognising that the sums in both the numerator and the denominator are over all phase space volumes defined by some $\Gamma^{\prime}$, where $B\left(S^{t_{1}} \Gamma^{\prime}\right)=A$, Eq. (9) can be rewritten as a conditional average

$$
\frac{\operatorname{Pr}\left(B\left(S^{t_{1}} \boldsymbol{\Gamma}\right)=A\right)}{\operatorname{Pr}\left(B\left(S^{t_{1}} \boldsymbol{\Gamma}\right)=-A\right)}=\frac{1}{\left\langle e^{-\Omega_{2 t_{1}}(\boldsymbol{\Gamma} ; 0)}\right\rangle_{B\left(S^{t_{1}} \mathbf{\Gamma}\right)=A}} .
$$

Similarly,

$$
\frac{\operatorname{Pr}\left(B\left(S^{t_{1}} \boldsymbol{\Gamma}\right)=A\right)}{\operatorname{Pr}\left(B\left(S^{t_{1}} \boldsymbol{\Gamma}\right)=-A\right)}=\left\langle e^{-\Omega_{2 t_{1}}(\boldsymbol{\Gamma} ; 0)}\right\rangle_{B\left(S^{t_{1}} \boldsymbol{\Gamma}\right)=-A} .
$$

This relationship is an instantaneous fluctuation relation, written in terms of conditional ensemble averages of exponentials of the dissipation function path integrals. These path integrals are symmetrically extended both before and after the time at which the instantaneous fluctuation relation is required. This instantaneous fluctuation relation is non-local in time. This is a standard feature of fluctuation relations that are required at times greater than zero. ${ }^{17}$ Another example is the asymptotic steady state Evans-Searles fluctuation relation. ${ }^{21,23}$

We can look at the behavior of the relative probabilities of opposite values of $A$ as a function of time, $t_{1}$. Initially, when $t_{1}$ $=0$, the dissipation function $\Omega_{2 t_{1}}(\Gamma ; 0)=0$, and so the RHS of Eq. (11) is equal to 1 and both values of A are equally probable. At later times the value of the dissipation function changes, meaning the opposite values of $A$ are not equally probable, corresponding to the irreversibility of the system 


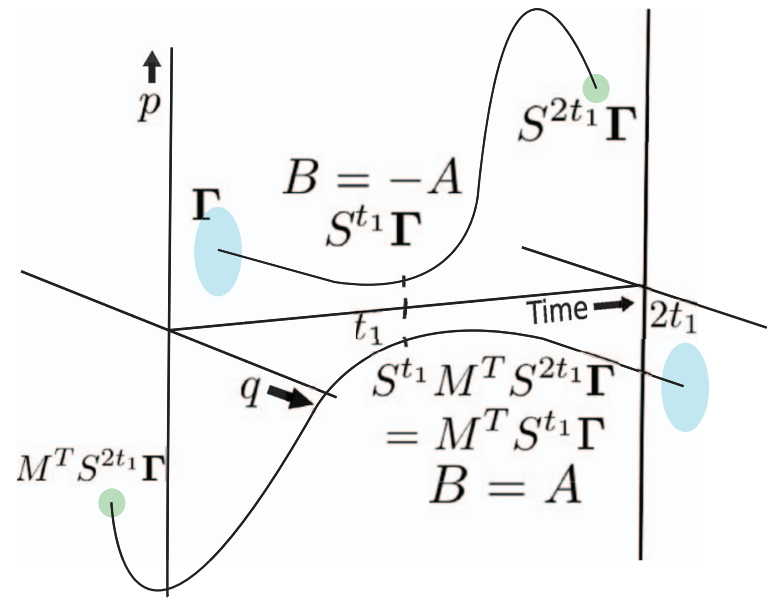

FIG. 1. A trajectory starting from $\Gamma$ with $B=-A$ at time $t=t_{1}$, along with its antitrajectory which begins from the time reversed map of $S^{2 t_{1}} \boldsymbol{\Gamma}$.

growing. If the system is T-mixing ${ }^{24}$ and there is a thermostatting mechanism in place then it must relax to a nonequilibrium ergodic steady state. ${ }^{25}$ We can look at the behavior of the new instantaneous fluctuation theorem in the steady state by first assuming that the dissipation function is delta correlated. As such we can write

$$
\begin{aligned}
\left\langle e^{-\Omega_{2 t_{1}}(\boldsymbol{\Gamma} ; 0)}\right\rangle_{B\left(S_{1}^{t_{1}} \boldsymbol{\Gamma}\right)=-A}= & \left\langle e^{-\Omega_{t_{1}}(\boldsymbol{\Gamma} ; 0)}\right\rangle\left\langle e^{-\Omega_{t_{1}}\left(S^{t_{1}} \boldsymbol{\Gamma} ; 0\right)}\right\rangle \\
& \times\left\langle e^{-\Omega_{0}\left(S^{t_{1}} \boldsymbol{\Gamma} ; 0\right)}\right\rangle_{B\left(S^{t_{1}} \boldsymbol{\Gamma}\right)=-A} .
\end{aligned}
$$

From the nonequilibrium partition identity NPI (formally referred to as the Kawasaki identity ${ }^{26,27}$ )

$$
\left\langle e^{-\Omega_{t_{1}}(\Gamma ; 0)}\right\rangle=1 \text {. }
$$

Also,

$$
\left\langle e^{-\Omega_{2 t_{1}}(\boldsymbol{\Gamma} ; 0)}\right\rangle=\left\langle e^{-\Omega_{t_{1}}(\boldsymbol{\Gamma} ; 0)}\right\rangle\left\langle e^{-\Omega_{t_{1}}\left(S_{1}^{t_{1}} \boldsymbol{\Gamma} ; 0\right)}\right\rangle
$$

but $\left\langle e^{-\Omega_{2 t_{1}}(\boldsymbol{\Gamma} ; 0)}\right\rangle=1$ and $\left\langle e^{-\Omega_{t_{1}}(\boldsymbol{\Gamma} ; 0)}\right\rangle=1$, so $\left\langle e^{-\Omega_{t_{1}}\left(S^{t_{1}} \Gamma ; 0\right)}\right\rangle$ $=1$. Now, from Eq. (12) we have

$$
\left\langle e^{-\Omega_{2 t_{1}}(\boldsymbol{\Gamma} ; 0)}\right\rangle_{B\left(S^{t_{1}}\right)=-A}=\left\langle e^{-\Omega_{0}\left(S^{t_{1}} \boldsymbol{\Gamma} ; 0\right)}\right\rangle_{B\left(S^{t_{1}}\right)=-A} .
$$

In a steady state this is time independent so the relative probability of $A$ to $-A$ is constant.

For a T-mixing system that is not delta correlated the correlations will decay to some arbitrarily fine tolerance for some time greater than some cut-off time, $c \tau_{M}$ (some multiple of the Maxwell time, $\tau_{M}$ ). Now the condition on the average affects the time interval $t_{1}-c \tau_{M}, t_{1}+c \tau_{M}$. There will be some correlation between this time interval and the surrounding time intervals, so if we separate the average into three parts as we did in Eq. (12) an error will be introduced. However, with a long $t_{1}$ in the T-mixing system the correlated part of the time intervals will be relatively small, making the error insignificant. ${ }^{23}$ Therefore, the instantaneous fluctuation theorem becomes

$$
\frac{\operatorname{Pr}\left(B\left(S^{t_{1}} \boldsymbol{\Gamma}\right)=A\right)}{\operatorname{Pr}\left(B\left(S^{t_{1}} \boldsymbol{\Gamma}\right)=-A\right)}=\left\langle e^{-\Omega_{2 c \tau_{M}}\left(S^{t_{1}-c \tau_{M}} \boldsymbol{\Gamma} ; 0\right)}\right\rangle_{B\left(S^{t_{1}} \boldsymbol{\Gamma}\right)=-A} .
$$

If the time $t_{1}-c \tau_{M}$ is in the steady state this expression will be time independent, and so the relative probability of opposite values of $A$ will be constant, as expected.
To those not used to the mathematical definition of dissipation the fact that $\Omega_{0}\left(S^{t_{1}} \boldsymbol{\Gamma} ; t_{1}\right) \neq 0$ may seem strange. It is due to the fact that in our work we always assume that the initial distribution is even in the momenta. This guarantees that the average velocity of the system is zero with respect to the observer and it also guarantees that $\Omega_{0}(\boldsymbol{\Gamma} ; 0)=0, \forall \boldsymbol{\Gamma}$, because $f(\boldsymbol{\Gamma} ; 0) / f\left(M^{T} \boldsymbol{\Gamma} ; 0\right)=1$. At later times, $f(\boldsymbol{\Gamma} ; t) / f\left(M^{T} \boldsymbol{\Gamma} ; t\right) \neq 1$.

We may also obtain an obvious summing rule from (10) and (11). Upon setting $A=0$ we obtain

$$
\left\langle e^{-\Omega_{2 t_{1}}(\boldsymbol{\Gamma} ; 0)}\right\rangle_{B\left(S^{t_{1}} \mathbf{\Gamma}\right)=0}=1 .
$$

This may be understood in terms of ergodic consistency. When $B\left(S^{t_{1}} \boldsymbol{\Gamma}\right)=0$ the set of trajectories used to form the above average is the exact same set as the conjugate antitrajectories and thus we have ergodic consistency over this restricted set of phase space. The phase space volume at time 0 coincides with the phase space volume at time $2 t_{1}$, since the effect of $B$ being odd under time reversal symmetry is lost when we set $B\left(S^{t_{1}} \boldsymbol{\Gamma}\right)=0$. Because of this ergodic consistency the above summing rule may be viewed as an application of the NPI to this restricted set of phase space. The FT can also be applied to this subset of phase space.

\section{NUMERICAL RESULTS}

To get a better understanding of the convergence and nature of the new instantaneous fluctuation relation we studied it numerically with a simple system. We modeled shear flow in a constant kinetic energy system that started from the equilibrium isokinetic ensemble. We chose to look at this in a two-dimensional 32 particle system. An isokinetic thermostat was used to obtain the desired starting conditions and keep a constant kinetic energy throughout the simulation. The system is subject to a constant shear at time $t>0$ using the SLLOD equations of motion ${ }^{26,28}$ and square unit cell Lees-Edwards periodic boundary conditions. ${ }^{29}$ Particle interactions were modeled using the Weeks-Chandler-Andersen potential. $^{30}$ The simulations were run with reduced units. ${ }^{26}$ The temperature used was $T=1$, the density was $\rho=0.6$, and the time step used was $d t=0.001$. Transient trajectories of length $t_{1}=0.1$ were initiated from positions found in the equilibrium system and were propagated using the equations of motion with an applied strain rate of $\dot{\gamma}=0.1$.

In the analysis above we generate the antitrajectories using a time reversal map of the final point in a trajectory, and then evolve this point in time using the equations of motion. A time reversal map in our chosen system would change the sign of the strain rate, $\dot{\gamma}$. It is inconvenient and unnecessary to run the simulation with two values of $\dot{\gamma}$ because we can instead use a different trajectory mapping. The appropriate mapping is known as the Kawasaki mapping, ${ }^{26}\left(\mathbf{x}, \mathbf{y}, \mathbf{p}_{\mathbf{x}}, \mathbf{p}_{\mathbf{y}}, \dot{\gamma}\right)$ $\rightarrow\left(\mathbf{x},-\mathbf{y},-\mathbf{p}_{\mathbf{x}}, \mathbf{p}_{\mathbf{y}}, \dot{\gamma}\right)$. Evolving the new point generated from this mapping forward in time using a strain rate $\dot{\gamma}$ generates the same antitrajectory as evolving a time reversed map point forward in time with the strain rate $-\dot{\gamma}$. The analysis of this system is otherwise identical to the general case above.

In order to demonstrate the new fluctuation relation we need to select a phase function of the system that is odd under 
the Kawasaki mapping. We can then numerically calculate the relative probabilities of particular values of the phase function at a given time, and compare it to the integrated dissipation function calculated over twice this time. A convenient phase function to use is the dissipative flux

$$
V P_{x y}(\boldsymbol{\Gamma})=\sum_{i=1}^{N}\left[\frac{p_{y i} p_{x i}}{m}+F_{x i} y_{i}\right],
$$

where $p_{y i}$ and $p_{x i}$ are the components of the peculiar momenta of particle $i, F_{x i}$ is the $x$ component of force on particle $i, y_{i}$ is the $y$ component of the position and in our 2D system and $V$ is the area of the unit cell. Rewritten for easier computation in a system which has periodic boundary conditions this becomes

$$
V P_{x y}(\boldsymbol{\Gamma})=\sum_{i=1}^{N} \frac{p_{y i} p_{x i}}{m}-\frac{1}{2} \sum_{i, j=1}^{N} F_{x i j} y_{i j},
$$

where $F_{x i j}$ is the force on particle $i$ due to $j$ and $y_{i j} \equiv y_{j}-y_{i}$.

To demonstrate the new fluctuation relation we need to calculate the relative probability of positive and negative values of $V P_{x y}\left(S^{t} \boldsymbol{\Gamma}\right)$ at each time we are interested in. This was done by constructing frequency histograms from an ensemble of trajectories, dividing the values of $V P_{x y}\left(S^{t_{1}} \boldsymbol{\Gamma}\right)$ into discrete bins and taking the ratio of frequencies for a positive valued bin to the corresponding negative bin. The integrated dissipation function, $\Omega_{2 t_{1}}(\boldsymbol{\Gamma} ; 0)$, was calculated for each time interval $2 t_{1}$. The following form of the dissipation function for this system is well known: ${ }^{1}$

$$
\Omega_{2 t_{1}}(\boldsymbol{\Gamma} ; 0)=-\int_{0}^{2 t_{1}} d s \beta V P_{x y}\left(S^{s} \boldsymbol{\Gamma}\right) \dot{\gamma}
$$

We calculated its value over the time interval $2 t_{1}$ and calculated the conditional average, $\left\langle e^{-\Omega_{2 t_{1}}(\Gamma ; 0)}\right\rangle_{V P_{x y}\left(S_{1} \boldsymbol{I}_{1}\right)=A}^{-1}$, based on the value of $V P_{x y}\left(S^{t_{1}} \boldsymbol{\Gamma}\right)$. The relative probability was plotted against the conditional average, seen in Figure 2(a). The uncertainties given in the equations in Figure 2 are the standard errors from the least squares fit of the data. To the order of the standard error, the slope of this graph is 1, demonstrating the new fluctuation relation. It was observed that the slope of Figure 2(a) trended upwards towards 1 with an increasing number of ensemble members used in the average. The system displays non-Gaussian statistics, a common feature in other non-equilibrium systems ${ }^{31-36}$ and non-equilibrium averages such as the non-equilibrium partition identity, ${ }^{26}$ and there is no known method to accurately determine the uncertainty in these calculations. In Figures 2(a) and 2(b) we also demonstrated the equivalence of the two forms of the instantaneous fluctuation theorem, Eqs. (10) and (11).

Since the form of this new fluctuation relation is not necessarily intuitive, it is instructive to look at the behavior of an altered form of the relation. We can consider the case where the dissipation function is integrated only up to the time where the relative probabilities of the phase functions are measured, i.e., over the time interval 0 to $t_{1}$. This simulation was conducted in the same manner as above and the results are included in Figure 2(c). The fluctuation relation is clearly not satisfied in this case as the slope of the curve is not 1 . This demonstrates that the use of conjugate sets of trajectories and

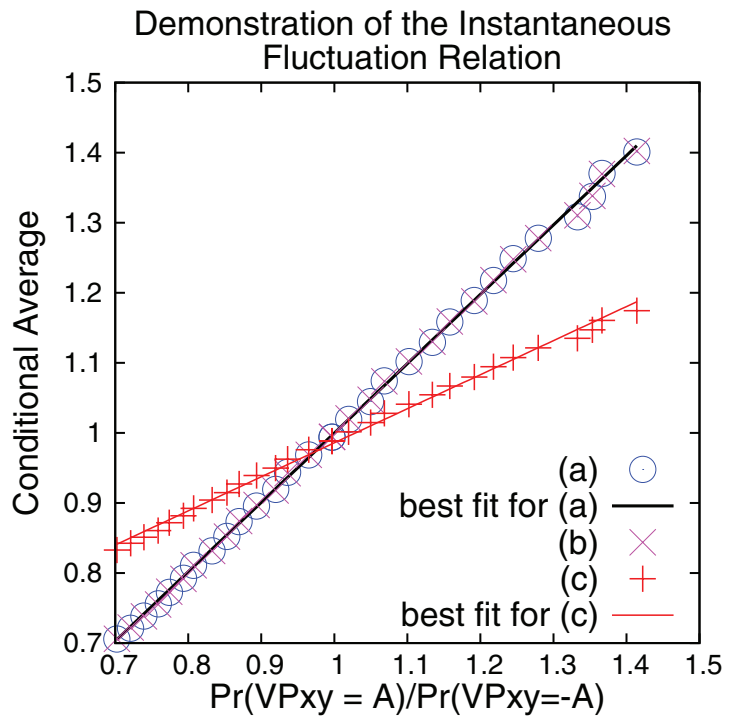

FIG. 2. (a) $\left\langle e^{-\Omega_{2 t_{1}}(\boldsymbol{\Gamma} ; 0)}\right\rangle_{V P_{x y}\left(S^{t_{1}} \mathbf{\Gamma}\right)=A}^{-1}$, demonstration of Eq. (10) with a line of best fit $y=(0.994 \pm 0.001) x+(0.006 \pm 0.001)$. (b) $\left\langle e^{-\Omega_{2 t_{1}}(\Gamma ; 0)}\right\rangle_{V P_{x y}\left(S^{t} 1 \Gamma\right)=-A}$, demonstration of Eq. (11), the second equivalent form of the relation. Note that the crosses are falling on top of the circles. (c) $\left\langle e^{-\Omega_{t_{1}}(\boldsymbol{\Gamma} ; 0)}\right\rangle_{V P_{x y}\left(S^{t_{1}} \Gamma\right)=-A}$, test of altered form of the relation with a line of best fit $y=(0.488 \pm 0.003) x+(0.497 \pm 0.003)$.

antitrajectories is essential in this fluctuation relation, as it is in the original Evans-Searles fluctuation relation. ${ }^{37}$

To prove that this result is not unique to the dissipative flux we demonstrated the new theorem using the phase functions $\sum_{i=1}^{N} p_{y i} p_{x i} / m$ and $-\frac{1}{2} \sum_{i, j=1}^{N} F_{x i j} y_{i j}$. These functions are the kinetic and configurational components of the dissipative flux, and so are clearly different from it. In both cases the new instantaneous fluctuation relation is satisfied, as seen in Figure 3.

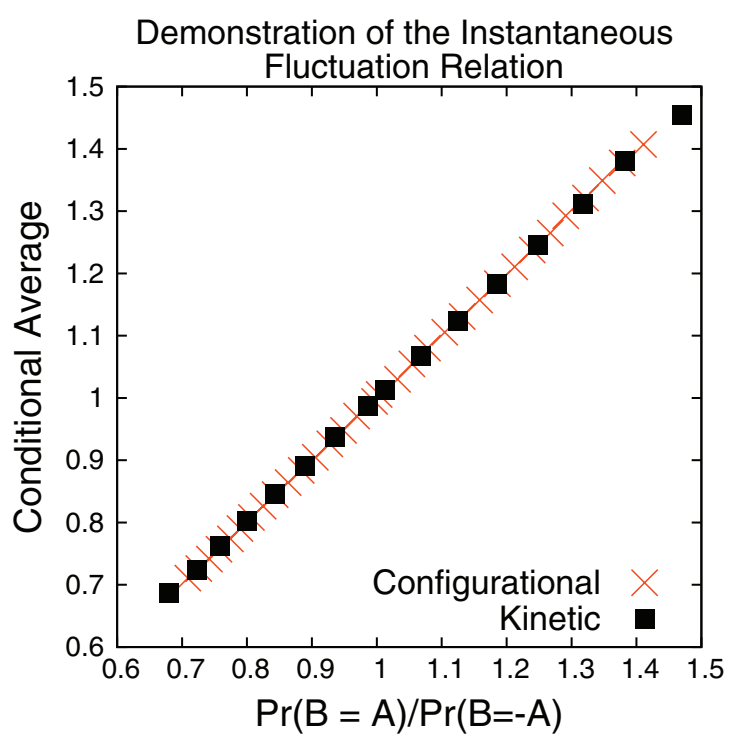

FIG. 3. Demonstration of the instantaneous fluctuation relation using the kinetic and configurational components of the dissipative flux. The equations of best fit are $y=(0.981 \pm 0.002) x+(0.019 \pm 0.002)$ and $y=(0.9956$ $\pm 0.0009) x+(0.0042 \pm 0.0009)$, respectively. 


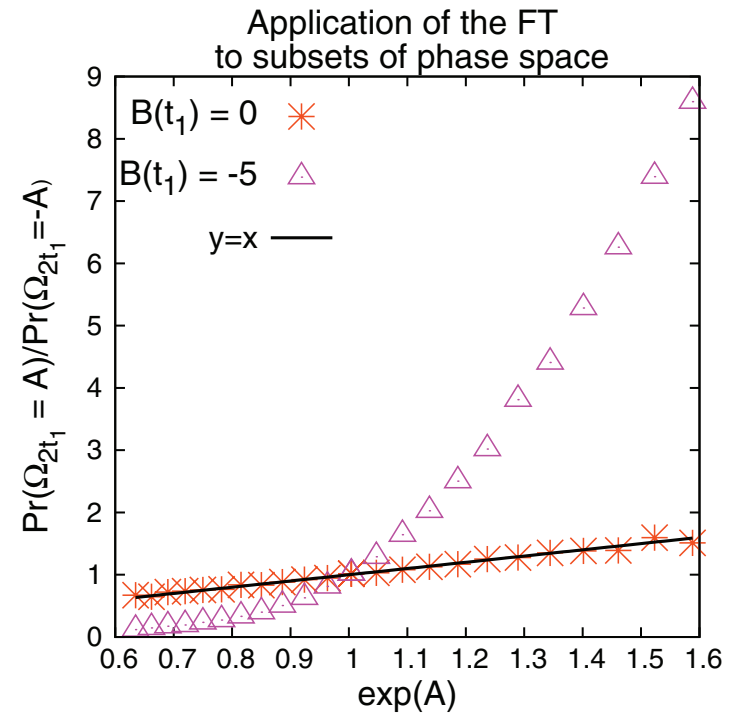

FIG. 4. Evans-Searles FT applied to two subsets of phase space, defined by $V P_{x y}\left(S^{t_{1}} \boldsymbol{\Gamma}\right)=0$ and $V P_{x y}\left(S^{t_{1}} \boldsymbol{\Gamma}\right)=-5$.

Ergodically consistent subsets of phase space can be defined by $B\left(S^{t_{1}} \boldsymbol{\Gamma}\right)=0$. The Evans-Searles FT can be applied to these subsets of phase space

$$
\frac{\operatorname{Pr}\left(\Omega_{2 t_{1}}(\boldsymbol{\Gamma} ; 0)=A\right)_{B\left(S^{t_{1}} \boldsymbol{\Gamma}\right)=0}}{\operatorname{Pr}\left(\Omega_{2 t_{1}}(\boldsymbol{\Gamma} ; 0)=-A\right)_{B\left(S^{t_{1}} \boldsymbol{\Gamma}\right)=0}}=\exp [A]
$$

where the trajectories included in the probability calculation are only those with $B\left(S^{t_{1}} \boldsymbol{\Gamma}\right)=0$. Using the same system as above, and the phase space function $B=V P_{x y}$, we can demonstrate the Evans-Searles FT in this subset of phase space.

Trajectories satisfying the criteria $V P_{x y}\left(S^{t_{1}} \boldsymbol{\Gamma}\right)=0$ were selected, and the dissipation function was calculated over the interval $\left(0,2 t_{1}\right)$. We demonstrated the FT in the usual way, by plotting the LHS of Eq. (21) against the RHS, seen in Figure 4 . The linear curve with a slope of 1 demonstrates the fluctuation theorem. To demonstrate that the FT is not applicable to all subsets of phase space, we carried out the procedure using trajectories where $V P_{x y}\left(S^{t_{1}} \boldsymbol{\Gamma}\right)=-5$ and plotted this in the same graph. This value for the dissipative flux was selected because it has a similar probability to $V P_{x y}\left(S^{t_{1}} \boldsymbol{\Gamma}\right)=0$ and so comparable statistics will be obtained. The attempted demonstration curve is clearly not linear, so the FT is not applicable to this non-ergodic subset of phase space.

\section{CONCLUSION}

We have derived and demonstrated computationally, a new fluctuation relation applicable to physical systems, which gives different information to those studied in the past. The instantaneous fluctuation relation is similar in form to other fluctuation relations, except that it considers path integrals of dissipation that are symmetrically extended both before and after the time at which the relative probabilities are compared. This work confirms the non time-local nature of dissipation. We also demonstrated that time reversibility for the trajectory/antitrajectory sets is a necessary condition for the fluctuation relation to hold. The instantaneous fluctuation relation shows how in driven, thermostatted, T-mixing systems, irreversibility (as manifest in the relative probabilities of odd phase functions taking on opposite values) grows as they approach nonequilibrium steady states.

${ }^{1}$ D. J. Evans and D. J. Searles, Adv. Phys. 51, 1529 (2002).

${ }^{2}$ E. M. Sevick, R. Prabhakar, S. R. Williams, and D. J. Searles, Annu. Rev. Phys. Chem. 59, 603 (2008).

${ }^{3}$ G. E. Crooks, J. Stat. Phys. 90, 1481 (1998).

${ }^{4}$ G. E. Crooks, Phys. Rev. E 60, 2721 (1999).

${ }^{5}$ G. Gallavotti and E. G. D. Cohen, Phys. Rev. Lett. 74, 2694 (1995).

${ }^{6}$ G. Gallavotti and E. G. D. Cohen, J. Stat. Phys. 80, 931 (1995).

${ }^{7}$ G. Gallavotti, Math. Phys. Electron. J. 1, 1 (1995).

${ }^{8}$ G. Gallavotti, Physica D: Nonlinear Phenom. 105, 163 (1997).

${ }^{9}$ G. Gallavotti, Physica A: Stat. Mech. Applic. 263, 39 (1999).

${ }^{10}$ G. Gallavotti, L. Rondoni, and E. Segre, Physica D: Nonlinear Phenom. 187, 338 (2004).

${ }^{11}$ J. Kurchan, J. Phys. A 31, 3719 (1998).

12 J. Lebowitz and H. Spohn, Journal of Statistical Physics 95, 333 (1999).

${ }^{13}$ C. Maes, Journal of Statistical Physics 95, 367 (1999).

${ }^{14}$ L. Rondoni, T. Tél, and J. Vollmer, Phys. Rev. E 61, R4679 (2000).

${ }^{15}$ R. van Zon and E. Cohen, Phys. Rev. Lett. 91, 110601 (2003).

${ }^{16} \mathrm{R}$. van Zon and E. Cohen, Phys. Rev. E 69, 056121 (2004).

${ }^{17}$ D. J. Evans, D. J. Searles, and S. R. Williams, J. Chem. Phys. 133, 054507 (2010).

${ }^{18}$ D. J. Searles and D. J. Evans, J. Chem. Phys. 113, 3503 (2000).

${ }^{19}$ D. J. Evans, D. J. Searles, and S. R. Williams, J. Chem. Phys. 128, 014504 (2008).

${ }^{20}$ D. J. Evans, D. J. Searles, and S. R. Williams, J. Stat. Mech.: Theory Exp. (2009) P07029.

${ }^{21}$ D. J. Searles, L. Rondoni, and D. J. Evans, J. Stat. Phys. 128, 1337 (2007).

${ }^{22}$ W. Hoover, O. Kum, and H. Posch, Phys. Rev. E 53, 2123 (1996).

${ }^{23}$ D. J. Searles, B. M. Johnston, D. J. Evans, and L. Rondoni, Entropy 15, 1503 (2013).

${ }^{24}$ D. J. Evans, D. J. Searles, and S. R. Williams, J. Chem. Phys. 132, 024501 (2010).

${ }^{25}$ D. J. Evans, S. R. Williams, D. J. Searles, and L. Rondoni, "On the relaxation to nonequilibrium steady states" (unpublished).

${ }^{26}$ D. J. Evans and G. P. Morriss, Statistical Mechanic of Nonequilibrium Liquids, 1st ed. (Academic Press, London, 1990).

${ }^{27}$ D. M. Carberry, S. R. Williams, G. M. Wang, E. M. Sevick, and D. J. Evans, J. Chem. Phys. 121, 8179 (2004).

${ }^{28}$ P. J. Daivis and B. D. Todd, J. Chem. Phys. 124, 194103 (2006).

${ }^{29}$ A. W. Lees and S. F. Edwards, J. Phys. C: Solid State Phys. 5, 1921 (1972).

${ }^{30}$ J. Weeks, D. Chandler, and H. Andersen, J. Chem. Phys. 54, 5237 (1971).

${ }^{31}$ L. Rondoni and E. Segre, Nonlinearity 12, 1471 (1999).

${ }^{32}$ J. Reid, D. Carberry, G. Wang, E. Sevick, D. Evans, and D. Searles, Phys. Rev. E 70, 016111 (2004)

${ }^{33}$ D. Collin, F. Ritort, C. Jarzynski, S. B. Smith, I. Tinoco, and C. Bustamante, Nature (London) 437, 231 (2005).

${ }^{34}$ V. Blickle, T. Speck, L. Helden, U. Seifert, and C. Bechinger, Phys. Rev. Lett. 96, 070603 (2006).

${ }^{35}$ K. Feitosa and N. Menon, Phys. Rev. Lett. 92, 164301 (2004).

${ }^{36}$ S. Ciliberto and C. Laroche, J. Phys. IV 8, Pr6 (1998).

${ }^{37}$ E. Mittag, D. J. Evans, and S. R. Williams, Pure Appl. Chem. 79, 1361 (2007). 